\title{
Realization of a Density-Dependent Peierls Phase in a Synthetic, Spin-Orbit Coupled Rydberg System
}

\author{
Vincent Lienhard, ${ }^{1, *}$ Pascal Scholl, ${ }^{1, *}$ Sebastian Weber $\odot,{ }^{2}$ Daniel Barredo $\odot,{ }^{1}$ Sylvain de Léséleuc $\odot,{ }^{1}$ Rukmani Bai, ${ }^{2}$ \\ Nicolai Lang $\odot,{ }^{2}$ Michael Fleischhauer, ${ }^{3}$ Hans Peter Büchler $\odot,{ }^{2}$ Thierry Lahaye $\odot,{ }^{1}$ and Antoine Browaeys $\circledast^{1}$ \\ ${ }^{1}$ Université Paris-Saclay, Institut d'Optique Graduate School, CNRS, Laboratoire Charles Fabry, \\ 91127 Palaiseau Cedex, France \\ ${ }^{2}$ Institute for Theoretical Physics III and Center for Integrated Quantum Science and Technology, \\ University of Stuttgart, 70550 Stuttgart, Germany \\ ${ }^{3}$ Department of Physics and Research Center OPTIMAS, University of Kaiserslautern, \\ 67663 Kaiserslautern, Germany
}

(Received 28 January 2020; accepted 19 March 2020; published 8 May 2020)

\begin{abstract}
We experimentally realize a Peierls phase in the hopping amplitude of excitations carried by Rydberg atoms, and observe the resulting characteristic chiral motion in a minimal setup of three sites. Our demonstration relies on the intrinsic spin-orbit coupling of the dipolar exchange interaction combined with time-reversal symmetry breaking by a homogeneous external magnetic field. Remarkably, the phase of the hopping amplitude between two sites strongly depends on the occupancy of the third site, thus leading to a correlated hopping associated with a density-dependent Peierls phase. We experimentally observe this density-dependent hopping and show that the excitations behave as anyonic particles with a nontrivial phase under exchange. Finally, we confirm the dependence of the Peierls phase on the geometrical arrangement of the Rydberg atoms.
\end{abstract}

DOI: $10.1103 /$ PhysRevX.10.021031

Subject Areas: Atomic and Molecular Physics,

Quantum Physics

\section{INTRODUCTION}

Synthetic quantum systems, i.e., well-controlled systems of interacting particles, are appealing to study many-body phenomena inspired by condensed matter physics [1]. One of the current challenges using this approach is to investigate the interplay between the nontrivial topology of a band structure, resulting from, e.g., an effective magnetic field, and the interactions between the particles $[2,3]$.

An effective magnetic field can be simulated by implementing complex hopping amplitudes $t e^{i \varphi}$ between the sites of an array, characterized by a Peierls phase $\varphi$ [4-6]. A particle circulating around a closed loop then acquires a phase analog to the Aharonov-Bohm phase, which is proportional to the enclosed magnetic flux. Effective magnetic fields and complex-valued hopping amplitudes have been implemented on ultracold atom-based platforms [3,7-10], by using laser-assisted tunneling in an optical superlattice [11], high-frequency driving of a lattice [12-14], and implementing synthetic dimensions [15-17]. Alternative platforms have

\footnotetext{
"These authors contributed equally to this work.
}

Published by the American Physical Society under the terms of the Creative Commons Attribution 4.0 International license. Further distribution of this work must maintain attribution to the author(s) and the published article's title, journal citation, and DOI. also emerged such as superconducting qubits where complexvalued hopping amplitudes were demonstrated [18], and photonic [19] or phononic [20] systems operating so far in the noninteracting regime. Here, we present the experimental realization of Peierls phases using the intrinsic spin-orbit coupling present in dipolar exchange interactions between Rydberg atoms.

Platforms involving individual Rydberg atoms are promising candidates to realize strongly interacting synthetic quantum matter [21,22]. The assembly of up to around 100 atoms in tunable geometries has already been achieved [23-28]. The two different regimes of interaction, van der Waals and resonant dipole-dipole [29], have been used, respectively, to implement Ising-like [30-32] or $X Y$ spin Hamiltonians $[33,34]$. In the resonant dipole-dipole regime, when the Rydberg atoms can be considered as two-level systems with states $n S$ and $n P$, the interaction results in the hopping of the $n P$ excitation between two sites, making it possible to explore transport phenomena. We recently used this fact to realize a symmetry-protected topological phase for interacting bosons [34]. Going beyond this two-level configuration, it has been proposed to engineer situations where the effective particle features an internal degree of freedom. There, the dipole-dipole interaction couples this internal degree of freedom with the motional one, resulting in an intrinsic spin-orbit coupling [35]. In combination with breaking of the time-reversal symmetry, this can lead to 
topological band structures characterized by nonzero Chern numbers [36-38].

In this paper, we demonstrate this intrinsic spin-orbit coupling in a minimal setup of three Rydberg atoms in a triangle. A combination of static magnetic and electric fields perpendicular to the triangle allows us to isolate two levels in the $n P$ manifold, thus giving rise to an excitation with two internal states. The external magnetic field naturally breaks the time-reversal symmetry, which, combined with the spinorbit coupling, leads to a characteristic chiral motion for a single excitation. We experimentally demonstrate this chiral motion and show that the dynamics is reversed by inverting the direction of the magnetic field. The chiral motion is well understood in an effective description, where one internal state of the excitation is adiabatically eliminated. In this case, the effective Hamiltonian is described by a nontrivial Peierls phase $\varphi$ in the hopping amplitude, corresponding to a finite magnetic flux through the triangle. Remarkably, in this approach the Peierls phase depends on the absence or presence of a second excitation, and naturally gives rise to density-dependent hoppings, which are required for the creation of dynamical gauge fields [39], as recently realized for ultracold atoms in optical lattices [40-42]. Here, we demonstrate this density-dependent hopping by observing the absence of chiral dynamics for two excitations. Following [43], the density-dependent hopping can be mapped to a hard-core anyon model with a statistical exchange angle $3 \varphi$. Finally, we demonstrate the ability to tune the effective magnetic flux through the triangle by varying the geometrical arrangement of the three atoms. We conclude by discussing the implications of this spin-orbit coupling on square and honeycomb plaquettes.

\section{SPIN-ORBIT COUPLING USING DIPOLAR EXCHANGE INTERACTIONS}

Our system consists of three ${ }^{87} \mathrm{Rb}$ atoms trapped in optical tweezers placed in an equilateral configuration, see Fig. 1(a). For each atom, we consider three Rydberg states from the $60 S_{1 / 2}$ and the $60 P_{3 / 2}$ manifolds (separated in frequency by $17.2 \mathrm{GHz}$ ) in a $V$ structure, as shown in Fig. 1(b). The state $|0\rangle=\left|60 S_{1 / 2}, m_{j}=1 / 2\right\rangle$ corresponds to the absence of excitation, and the two excited states $|+\rangle=$ $\left|60 P_{3 / 2}, m_{j}=3 / 2\right\rangle$ and $|-\rangle=\left|60 P_{3 / 2}, m_{j}=-1 / 2\right\rangle$, correspond to the two internal states of the excitation. We describe these two components of the excitation on a site $i$ by the bosonic operators $a_{i}^{\dagger}$ and $b_{i}^{\dagger}$ defined by $a_{i}^{\dagger}|0\rangle=|+\rangle_{i}$ and $b_{i}^{\dagger}|0\rangle=|-\rangle_{i}$. The energy difference $\mu=E_{+}-E_{-}$ between $|+\rangle$ and $|-\rangle$ is controlled by a magnetic field $B_{z}$ and an electric field $E_{z}$, both orthogonal to the atomic array. The excitation transfer between two Rydberg atoms is governed by the dipole-dipole interaction $\hat{V}_{i j}=\left(\hat{\boldsymbol{d}}_{i} \cdot \hat{\boldsymbol{d}}_{j}-3\left(\hat{\boldsymbol{d}}_{i} \cdot \hat{\boldsymbol{r}}\right)\left(\hat{\boldsymbol{d}}_{j} \cdot \hat{\boldsymbol{r}}\right)\right) /\left(4 \pi \epsilon_{0} r_{i j}^{3}\right)$. In our configuration, the unit vector $\hat{\boldsymbol{r}}=(\cos \phi, \sin \phi, 0)$ lies in the $(x, y)$ plane, and $\hat{V}_{i j}$ thus reads (a)
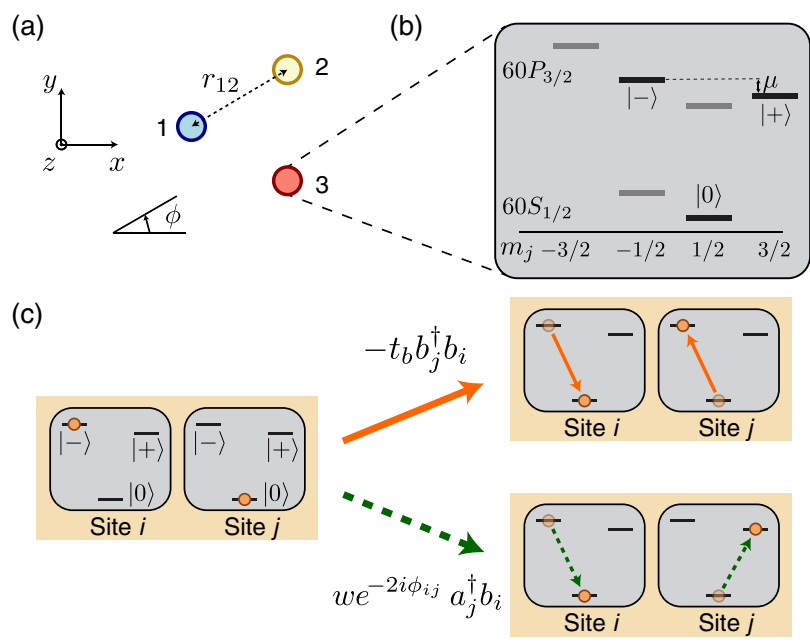

FIG. 1. Spin-orbit coupling induced by dipolar exchange interaction. (a) Experimental configuration of three atoms trapped in a tunable geometry. The quantization axis $z$, along the magnetic field, is perpendicular to the array of atoms. (b) Schematic Zeeman structure of the two Rydberg manifolds $60 S_{1 / 2}$ and $60 P_{3 / 2}$ used in this work. The three levels $|0\rangle,|+\rangle$, and $|-\rangle$ of the $V$ structure involved in the dipole-dipole interaction are indicated as black lines. The energy difference between $|+\rangle$ and $|-\rangle$ is $\mu$, controlled by dc magnetic and electric fields perpendicular to the triangle. (c) The two processes for a $|-\rangle$ excitation to hop from site $i$ to site $j$ : the $|-\rangle$ excitation is annihilated on site $i$, and a $|-\rangle$ (solid arrow) or a $|+\rangle$ (dashed arrow) excitation is created on site $j$.

$$
\begin{aligned}
\hat{V}_{i j}= & \frac{1}{4 \pi \epsilon_{0} r_{i j}^{3}}\left[\hat{d}_{i}^{z} \hat{d}_{j}^{z}+\frac{1}{2}\left(\hat{d}_{i}^{+} \hat{d}_{j}^{-}+\hat{d}_{i}^{-} \hat{d}_{j}^{+}\right)\right. \\
& \left.-\frac{3}{2}\left(\hat{d}_{i}^{+} \hat{d}_{j}^{+} e^{-i 2 \phi_{i j}}+\hat{d}_{i}^{-} \hat{d}_{j}^{-} e^{i 2 \phi_{i j}}\right)\right] .
\end{aligned}
$$

Here, $\hat{d}_{i}^{x}, \hat{d}_{i}^{y}, \hat{d}_{i}^{z}$ are the components of the dipole operator $\hat{\mathbf{d}}_{i}, \hat{d}_{i}^{ \pm}=\mp\left(\hat{d}_{i}^{x} \pm i \hat{d}_{i}^{y}\right) / \sqrt{2}$, and $r_{i j}$ and $\phi_{i j}$ denote the separation and the polar angle between the two Rydberg atoms. The first three terms in Eq. (1) correspond to a transfer of excitation conserving the total internal angular momentum of the two atoms. The last two terms describe the spin-orbit coupling: the excitation changes its internal state by two quanta during the transfer, and the conservation of the total angular momentum requires that the corresponding hopping amplitudes acquire a phase $e^{ \pm i 2 \phi_{i j}}$. Therefore, the dipolar interaction leads to two ways for an excitation to hop from site $i$ to site $j$, as illustrated in Fig. 1(c): a resonant process, with amplitude $-t_{a}$ or $-t_{b}$, where the internal state of the excitation is conserved, and an off-resonant process (by an energy offset $\mu$ ) with complex amplitude $w \mathrm{e}^{ \pm 2 i \phi_{i j}}$, where the excitation changes its internal state. The amplitudes $t_{a, b}$ and $w$ scale as $1 / r_{i j}^{3}$ (see more details in the Appendix A).

We now discuss the situation where three atoms are arranged in an equilateral triangle and derive the expression of the complex hopping amplitude of a $|-\rangle$ excitation. 
We restrict ourselves to the case $\mu \gg t_{a, b}, w$ and treat the hoppings perturbatively. As the internal state-flipping hopping is off resonant, the $|-\rangle$ excitation only has a small probability of becoming a $|+\rangle$ excitation. In addition, as the interaction conserves the number of excitations, once the atoms are initialized in the three-site state $|-00\rangle$, they mostly remain in the one excitation subspace consisting of the states $|-00\rangle,|0-0\rangle$, and $|00-\rangle$. The hopping of a $|-\rangle$ excitation from site 1 to 2 , i.e., the change of the three-atom state from $|-00\rangle$ to $|0-0\rangle$ [see Fig 2(a)], proceeds either by a direct hopping with amplitude $-t_{b}$, or by a secondorder coupling via the intermediate state $|00+\rangle$ consisting in two successive flips of the internal state. The latter has an amplitude $-w^{2} \mathrm{e}^{2 i\left(\phi_{32}-\phi_{13}\right)} / \mu$, with $\phi_{32}-\phi_{13}=2 \pi / 3$. Consequently, the hopping amplitude $-t e^{i \varphi}$ from site 1 to 2 is the sum of the amplitudes of these two processes

$$
t e^{i \varphi}=t_{b}+e^{i 4 \pi / 3} \frac{w^{2}}{\mu} .
$$

The representation of the amplitudes in the complex plane is shown in Fig. 2(b). In this perturbative picture, the $|+\rangle$ excitation is adiabatically eliminated, and the problem reduces to the hopping of the $|-\rangle=b_{i}^{\dagger}|0\rangle$ excitation described by the effective Hamiltonian

$$
H_{\mathrm{eff}}=-t \sum_{i=1}^{3}\left[e^{i \varphi} b_{i+1}^{\dagger} b_{i}+e^{-i \varphi} b_{i}^{\dagger} b_{i+1}\right]
$$

with $b_{4} \equiv b_{1}$. Here, we write the Hamiltonian in the second-quantized form in view of the extension to the multiexcitation case (see Sec. IV). The Peierls phase $\varphi$ can

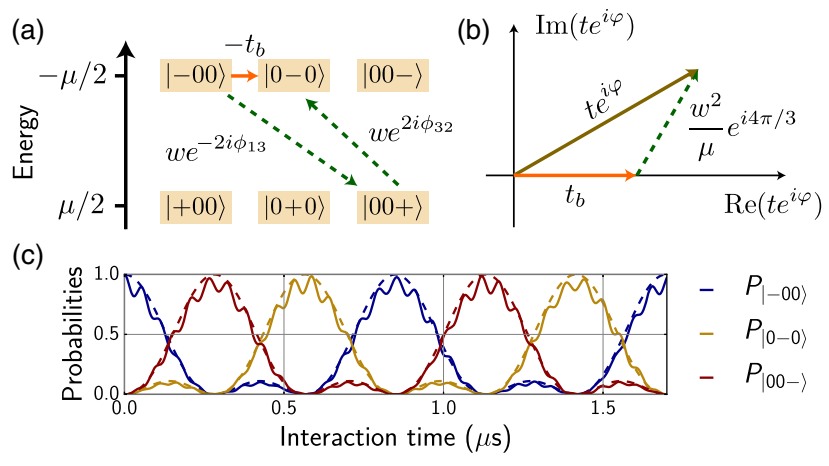

FIG. 2. Peierls phase on a triangle. (a) The two available processes for a $|-\rangle$ excitation to hop from $|-00\rangle$ to $|0-0\rangle$ : direct hopping with amplitude $-t_{b}$, or virtual hoppings via $|00+\rangle$. (b) Complex plane representation of the effective hopping, which is the sum of the two processes depicted in (a). (c) Calculated evolution of the site probabilities after preparing $|-00\rangle$ with total flux $3 \varphi=\pi / 2$, for an ideal complex hopping (dashed lines) and for our three-level structure involving the $|+\rangle$ states (solid lines). The excitation does not spread as time flows, and moves from site to site in a chiral way. be interpreted as the result of an emergent gauge field and the magnetic flux through the triangle is thus $3 \varphi$. Experimentally, both the effective hopping amplitude $t$ and the flux $3 \varphi$ are controlled by the distance between the atoms and the energy separation $\mu$. For nonzero flux (modulo $\pi$ ), the excitation exhibits a chiral motion when evolving in the triangle. In particular, for $3 \varphi= \pm \pi / 2$ [18], the excitation hops sequentially from site to site in a preferred direction. Figure 2(c) shows this expected motion for the parameters used in the experiment (see Sec. III): we plot the site probabilities as a function of time in the case of the complex hopping of a $|-\rangle$ excitation described by the Hamiltonian (3) (dashed lines), as well as for the three-level structure involving the $|+\rangle$ state, governed by the Hamiltonian (A1) (solid lines). The fast oscillations exhibit a frequency close to $\mu / h$, and result from the nonperfect elimination of the $|+\rangle$ state.

\section{EXPERIMENTAL OBSERVATION OF CHIRAL MOTION}

To experimentally demonstrate the chiral motion of a $|-\rangle$ excitation resulting from the complex hopping of Eq. (2), we start with three ${ }^{87} \mathrm{Rb}$ atoms trapped in 852-nm optical tweezers arranged in an equilateral triangle with a side length $11 \mu \mathrm{m}$ [24]. We optically pump the atoms in the state $\left|5 S_{1 / 2}, F=2, m_{y}=-2\right\rangle$ in $200 \mu$ s using a quantization axis defined by a magnetic field $B_{y}>0$ along the $y$ axis contained in the triangle plane. To isolate the $V$ structure in the Rydberg manifold and achieve isotropic exchange terms $t_{a, b}$ and $w$, we must apply static magnetic and electric fields perpendicular to the plane of the triangle. To do so, we switch on adiabatically $B_{z}$ and turn off $B_{y}$ after the optical pumping step, in $20 \mathrm{~ms}$ : for $B_{z}<0$ the resulting atomic state is thus $\left|5 S_{1 / 2}, F=2, m_{z}=2\right\rangle$. In order to fix the Peierls phase to the value leading to the chiral motion $(3 \varphi \approx \pi / 2)$, we set $B_{z}=-8.5 \mathrm{G}$ and the electric field $E_{z}=0.4 \mathrm{~V} / \mathrm{cm}$, yielding $\mu / h=-16 \mathrm{MHz}$. With these values and $r_{i j}=11 \mu \mathrm{m}$, we measure, from a spin exchange experiment with two atoms [33], $t_{a} / h \simeq 1.5 \mathrm{MHz}$, and $t_{b} / h \simeq 0.55 \mathrm{MHz}$ in good agreement with theoretical calculations of the interaction energies [44]. We then deduce $w / h \simeq 2.7 \mathrm{MHz}$ using the values of the angular part of the dipole matrix elements. After switching off the dipole traps, we prepare the $|000\rangle$ state in $2 \mu \mathrm{s}$ using a stimulated Raman adiabatic passage [34], via the intermediate state $\left|5 P_{1 / 2}, F=2, m_{z}=2\right\rangle$. Finally, we address atom 1 with a focused laser beam tuned near the $6 P_{3 / 2}-$ $60 S_{1 / 2}$ transition [45] and apply a 400-ns $\pi$ pulse with a microwave resonant with the light shifted $|0\rangle \rightarrow|-\rangle$ transition. This prepares a $|-\rangle$ excitation on site 1 .

After the preparation of the system in the state $|-00\rangle$, we let it evolve under the action of the dipole-dipole interaction for a time $\tau$. We then apply a 400-ns read-out pulse to deexcite the atoms in $|0\rangle$ back to the $5 S_{1 / 2}$ manifold, and 
switch on the dipole traps again. Atoms in the $5 S_{1 / 2}$ state are recaptured, whereas atoms still in Rydberg states are lost. A final fluorescence image reveals, for each site, if the atom is in the $|0\rangle$ state (the atom is recaptured), or in another Rydberg state (the atom is lost). Our detection method does not distinguish between these other Rydberg states, including $|+\rangle$ and $|-\rangle$. We will denote the Rydberg states other than $|0\rangle$ as a single state $|1\rangle$. As the $|+\rangle$ subspace is hardly populated in our experiment, the loss of an atom corresponds mainly to its detection in the $|-\rangle$ state.

The result of this first experiment is presented in Fig. 3(a), where we plot the three-site probabilities to be in the states $|100\rangle,|010\rangle$, and $|001\rangle$ as a function of the interaction time $\tau$. As expected, we observe a chiral motion of a localized $|-\rangle$ excitation in the counterclockwise direction $1 \rightarrow 3 \rightarrow 2 \rightarrow 1$. This is the signature of an effective magnetic field acting on the hopping excitation, described by the Peierls phases. The fact that the three probabilities do not sum to 1 comes from the imperfect preparation of the state $|100\rangle$ and detection errors.

To reverse the direction of motion, we reverse the sign of $B_{z}$ after the optical pumping stage. The initial atomic state is now $\left|5 S_{1 / 2}, F=2, m_{z}=-2\right\rangle$. In this configuration, following the Rydberg excitation, the $V$ structure in the Rydberg manifold involves $|0\rangle=\left|60 S_{1 / 2}, m_{J}=-1 / 2\right\rangle$, $|+\rangle=\left|60 P_{3 / 2}, m_{J}=-3 / 2\right\rangle$, and $|-\rangle=\left|60 P_{3 / 2}, m_{J}=+1 / 2\right\rangle$. The value of $\mu$ remains unchanged, as the Stark shift only depends on $\left|m_{j}\right|$. The hopping of a $|-\rangle$ to a $|+\rangle$ excitation now corresponds to a decrease of the internal momentum by two quanta: the orbital phase factor is thus $e^{2 i \phi_{i j}}$, and the sign of the Peierls phase is changed. Figure 3(c) shows the same three-site probabilities as in Fig. 3(b) for this opposite direction of $B_{z}$. As expected, we now observe a chiral motion of the $|-\rangle$ excitation in the clockwise direction $1 \rightarrow 2 \rightarrow 3 \rightarrow 1$.

Finally, we compare the experimental data for the chiral motion in both directions with a theoretical model solving the Schrödinger equation for this three-atom system including all the Zeeman sublevels of the $60 S_{1 / 2}$ and $60 P_{3 / 2}$ manifold. In these simulations, the preparation and detection errors are included as well as shot-to-shot fluctuations in the atomic positions; the latter leads to small modifications of the coupling parameters for each shot. The details of these simulations are presented in Appendix A. The results are plotted as solid lines on the data in Figs. 3(a) and 3(b). In both situations, we obtain a good agreement with the model, which reproduces the frequency, the amplitude, and the damping of the chiral motion.

\section{DENSITY-DEPENDENT PEIERLS PHASE AND MAPPING TO ANYONS}

For ensembles of two-level atoms in resonant interaction, the excitations can be mapped onto hard-core bosons, a fact used in our previous work [34]. A natural question to ask in our present multilevel situation is the consequence of the hard-core constraint on the dynamics of the $|-\rangle$ excitations. In order to explore this experimentally, we now initialize the three-atom system with two $|-\rangle$ excitations on sites 2 and 3 , while site 1 is in state $|0\rangle$, thus preparing the threeatom state $|0--\rangle$. To do so we again use the addressing
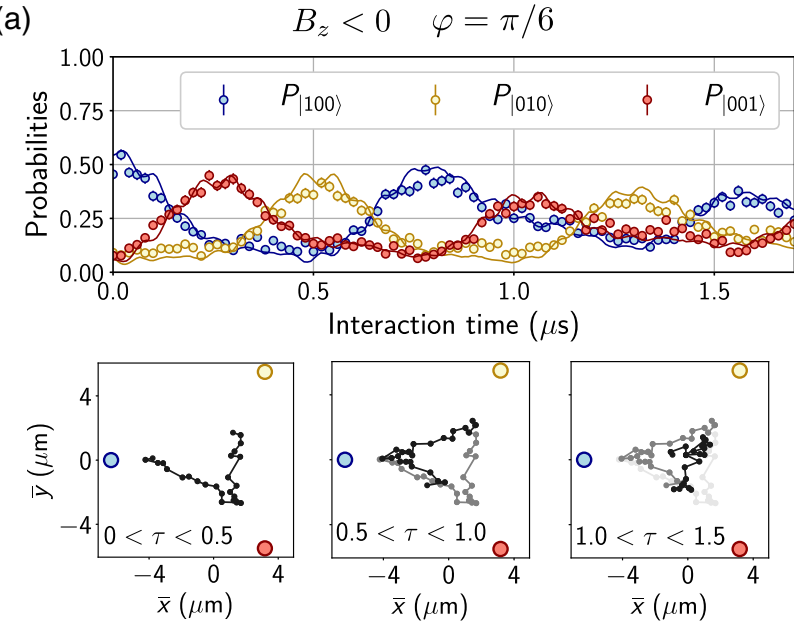

(b)
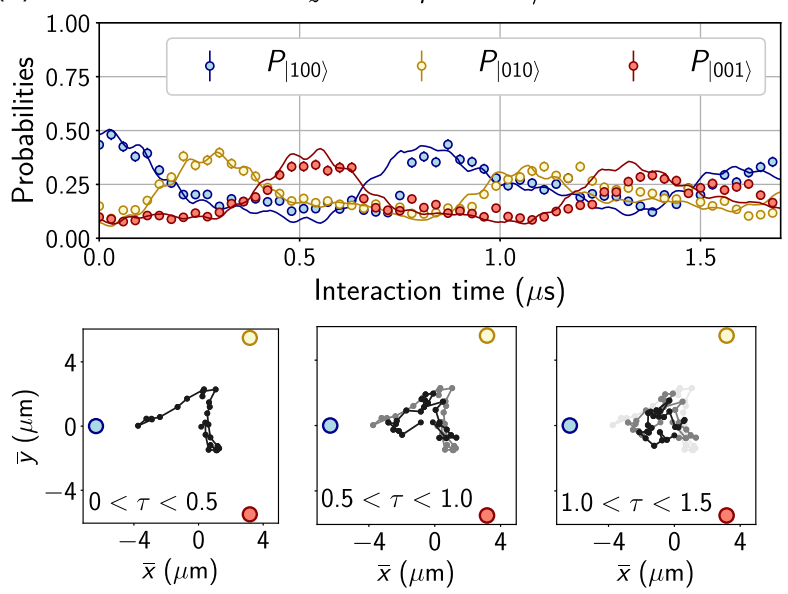

FIG. 3. Observation of the chiral motion of a single $|-\rangle$ excitation. (a),(b) Evolution of the three-site probabilities to be in the states $|100\rangle,|010\rangle$, and $|001\rangle$ as a function of the interaction time for two opposite directions of $B_{z}$. Upper panel: experimental results and theoretical predictions (solid lines) including experimental errors in the preparation and the detection, as well as shot-to-shot fluctuations in the atomic position (which lead to the observed damping of the oscillations). Bottom panel: associated trajectories of the center of mass of the excitation $(\bar{x}, \bar{y})$ for specific windows of the excitation time $\tau$, defined by $\bar{x}=\sum_{i=1}^{3} x_{i} p_{i} / \sum_{i=1}^{3} p_{i}$ and $\bar{y}=$ $\sum_{i=1}^{3} y_{i} p_{i} / \sum_{i=1}^{3} p_{i}$ [where $\left(x_{i}, y_{i}\right)$ are the coordinates of site $i$, and $p_{i}$ the probability for the $|-\rangle$ excitation to be on site $i$ ]. Error bars denote the standard error on the mean, and are often smaller than the symbol size. 
laser on site 1 , but tune the $\pi$ microwave pulse on resonance with the free-space $|0\rangle \rightarrow|-\rangle$ transition.

In the case of hard-core bosons evolving with the Hamiltonian in Eq. (3), one can show that the hole (state $|0\rangle$ ) propagates in the opposite direction with respect to the single $|-\rangle$ excitation case, as observed using superconducting circuits [18]. The result of our experiment is presented in Fig. 4, where we use the same parameters as for the single excitation experiment, i.e., a Peierls phase $\varphi=\pi / 6$. Remarkably, here we do not observe any chiral motion: the hole state $|0\rangle$ propagates almost symmetrically towards sites 2 and 3, suggesting that the hopping amplitude between sites is now real, and that the description of the dynamics by the Hamiltonian (3) is no longer valid. This indicates that the hard-core constraint between the excitations $|-\rangle$ influences the induced Peierls phases.

To understand this, we come back to the hard-core constraint in our system.

Two particles, irrespective of their internal state $|+\rangle$ or $|-\rangle$, cannot reside on the same site. As a consequence, the

(a)

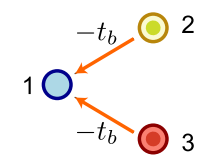

(b)
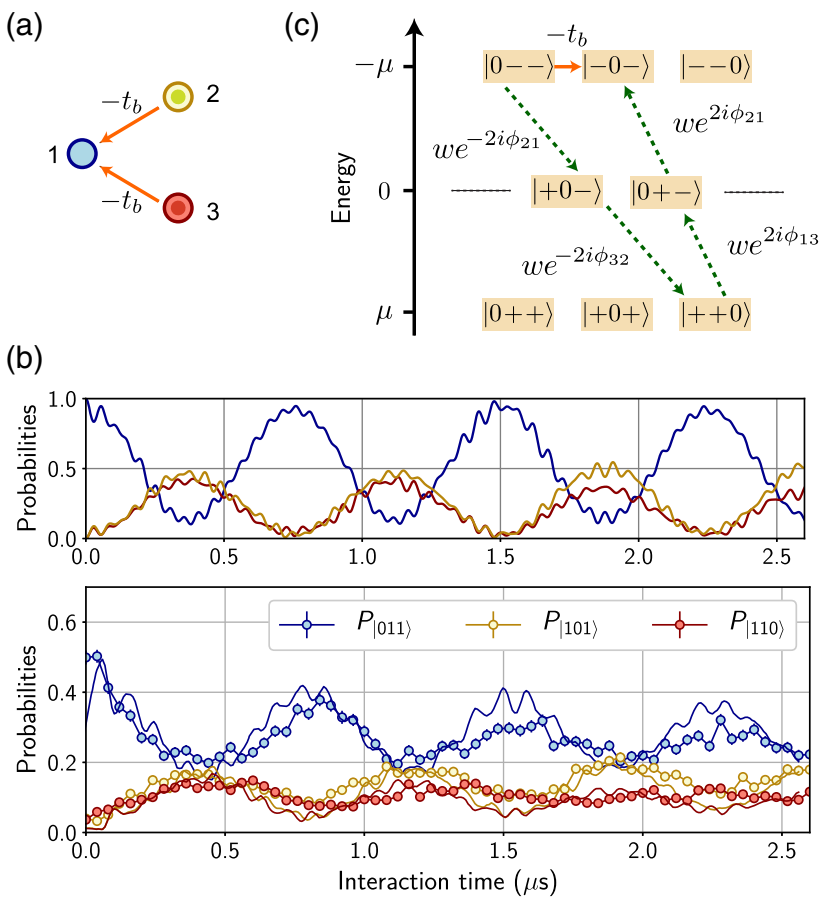

FIG. 4. Demonstration of density-dependent hopping for two excitations. (a) The presence of a $|-\rangle$ excitation on site 3 prevents the internal state-flipping process responsible for the complex hopping of the $|-\rangle$ excitation from 2 to 1 : only the real coupling remains. (b) Probability to be in the doubly excited three-site states $|011\rangle$ (targeted initial state), $|101\rangle$, or $|110\rangle$ as a function of the interaction time $\tau$. Upper panel: simulations in an ideal case including the three levels of the $V$ structure. Lower panel: experimental results together with the simulation taking into account experimental parameters, including state preparation. (c) Hopping processes to go from site 1 to site 2 in the twoexcitation case, showing the direct coupling and the fourth-order process via $|0++\rangle$. effective hopping from site 1 to 2 is modified if an excitation is already present on site 3: This suppresses the off-resonant process, which is at the origin of the complex hopping amplitude in the single excitation case, leaving only the direct hopping described by $-t_{b}$. Therefore, the hard-core constraint generates a densitydependent hopping, where the phase of the hopping amplitude, as well as its strength, depends on the occupation of the third lattice site. The effective Hamiltonian describing this situation generalizes the one of Eq. (3) to the case of more than one $|-\rangle$ excitation:

$H_{\mathrm{eff}}^{\mathrm{many}}=-t \sum_{i=1}^{3}\left[e^{i \varphi\left(1-n_{i+2}\right)} b_{i+1}^{\dagger} b_{i}+\Delta b_{i+1}^{\dagger} b_{i} n_{i+2}+\right.$ H.c. $]$,

with $n_{i+2}=b_{i+2}^{\dagger} b_{i+2}$ the occupation of the third site and $\Delta=\left(t_{b}-t\right) / t$. The first term in the effective Hamiltonian shows that the Peierls phase is now density dependent. The second term describes a conventional correlated hopping, which does not modify the real or complex nature of the couplings between sites (see Appendix B). In addition, the adiabatic elimination leads to two-body interaction terms $\propto\left(w^{2} / \mu\right) n_{i} n_{j}$, that do not play a role in an equilateral triangle and that we therefore drop.

The influence of the density-dependent Peierls phases on the hopping amplitudes has a simple interpretation in terms of Abelian anyonic particles in one dimension in the absence of a magnetic field [43,46-49]. Here, we obtain anyonic particles with a hard-core constraint and a statistical angle $3 \varphi$. For this mapping, we use a particle-hole transformation and interpret a single hole as an anyonic particle. In the absence of a gauge field, a single anyon (a hole) exhibits a symmetric dynamics in a triangle, which is the result observed in Fig. 4. Now placing two anyons (two holes) in the triangle, we are back to the case studied in Sec. III, where we observe a chiral motion (Fig. 3): In the anyon interpretation, this is due to the statistical phase under exchange of the two anyonic particles, or equivalently to the fact that one of the two anyonic particles carries a magnetic flux for the other one. The value of this magnetic flux through the triangle is the statistical phase of these anyons. The mapping onto anyons can be made rigorous and is presented in Appendix B.

We still observe a residual asymmetry in the dynamics, see Fig. 4(b), which is also present in the simulation. This indicates that the complex-valued hopping is not fully suppressed. Following the same effective Hamiltonian approach as the one outlined in Sec. II, the internal state-flipping hopping is now a fourth-order process, as shown in Fig. 4(c). Considering the hopping from site 1 to site 2 , the hole can directly hop with an amplitude $-t_{b}$, or virtually go through $|++0\rangle$, leading to a total amplitude $t e^{i \varphi}=t_{b}+w^{4} / \mu^{3} e^{-4 i \pi / 3}$. As $w \ll \mu$, the complex part of 
this hopping is extremely small compared to the single particle case, thus leading to the observed quasi-symmetric dynamics.

\section{TUNABILITY OF THE PEIERLS PHASE}

In a final experiment, we demonstrate the control of the Peierls phase in the single excitation case by tuning the geometry of the triangle while keeping the same value for $\mu$. To do so, we study an isosceles triangle parametrized by the angle $\gamma$, see Fig. 5(a). In this configuration, the distance between sites 1 and 3 varies with $\gamma$. The effective coupling, and hence the Peierls phase, is then different for each link: the direct hoppings are $t_{12}=t_{23}$ and $t_{13}=\kappa t_{12}$ with $\kappa=1 /(2 \cos [\gamma / 2])^{3}$; the virtual couplings are $\kappa w^{2} e^{i \gamma} / \mu$ for the $1 \rightarrow 2$ and $2 \rightarrow 3$ couplings and $w^{2} e^{-2 i \gamma} / \mu$ for the $3 \rightarrow 1$ coupling. The variation of the magnetic flux through the triangle, which is the sum of the three Peierls phases, is represented in Fig. 5(b) as a function of the angle $\gamma$. It exhibits an almost linear dependence for $\gamma \in\left[0^{\circ}, 90^{\circ}\right]$.

Our demonstration of the control over the Peierls phase is achieved by observing how a single $|-\rangle$ excitation prepared initially on site 2 splits between site 1 and site 3 after a given evolution time: for a negative flux (modulo $2 \pi$ ) the excitation propagates towards site 1 , while it propagates towards site 3 for a positive flux. For zero flux (modulo $\pi$ ) the propagation is symmetric. Figure 5(c) shows the result of the experiment. We plot the population imbalance between site 1 and site $3, \mathcal{I}=\left(P_{|100\rangle}-P_{|001\rangle}\right) /\left(P_{|001\rangle}+P_{|100\rangle}\right)$, at time $\tau=0.4 \mu \mathrm{s}$, as a function of the angle $\gamma$. We chose $\tau=0.4 \mu \mathrm{s}$ as it corresponds to the excitation mainly located on sites

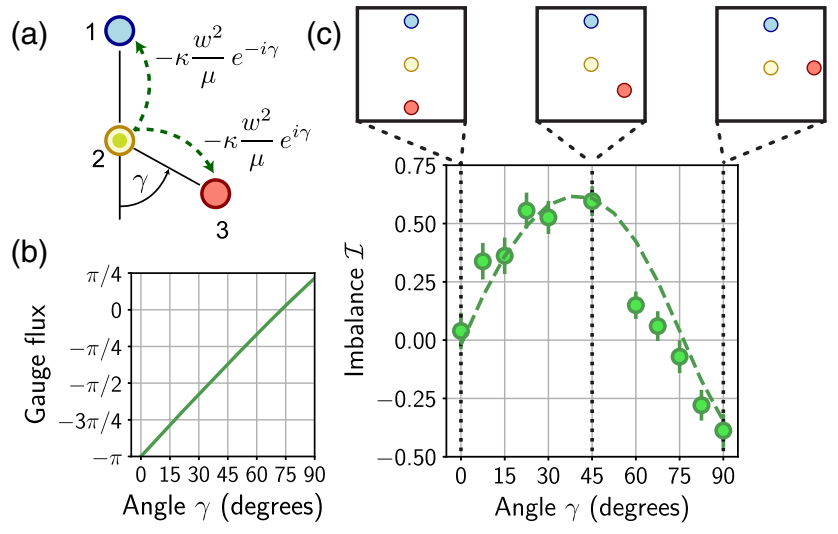

FIG. 5. Tunability of the Peierls phase. (a) Tunable geometry used for this experiment based on an isosceles triangle with $r_{12}=r_{23}=11 \mu \mathrm{m}$. (b) Calculated evolution of the magnetic flux threading through the isosceles triangle as a function of $\gamma$. (c) Experimental imbalance $\mathcal{I}$ between site 1 and site 3 (see text) after having prepared an excitation on site 2 and letting the system evolve for $\tau=0.4 \mu \mathrm{s}$, as a function of the angle $\gamma$. A positive imbalance means that the excitation mainly resides on site 1 . The three insets represent the triangle configurations for three values of $\gamma$, marked on the graph by the three dotted lines. The dashed line is the simulation.
1 and 3 for $\gamma=0^{\circ}$. As expected, we observe that the imbalance varies with the angle $\gamma$, and hence with the magnetic flux [Fig. 5(b)]. For $\gamma=0^{\circ}$ and $75^{\circ}$ (zero flux) the propagation is symmetric. The data are in good agreement with the simulation of the dynamics of the system (dashed line).

\section{EXTENSION TO OTHER GEOMETRIES}

As demonstrated in the previous section for the case of an isosceles triangle, the Peierls phase depends on the geometrical arrangement of the atoms. A natural question to ask is what happens for geometries other than a triangle. In the following, we discuss theoretically the Peierls phase patterns for plaquettes of square and honeycomb lattices, considering the perturbative regime where the $|+\rangle$ excitation can be eliminated.

For a square geometry, see Fig. 6(a), we find a nearest neighbor hopping $t e^{i \varphi}$ with a Peierls phase $\varphi$, as the adiabatic elimination gives rise to two distinct virtual processes of equal strength. On the contrary, the next-nearest-neighbor hopping remains real valued. Consequently, a single excitation experiences a homogeneous gauge field with a flux $4 \varphi$ through the square. As for the triangle case, the presence of a second excitation gives rise to a density-dependent hopping and quenches the virtual processes. Note that in addition to the density-dependent phase one needs to include in this case density-density interaction terms. These terms are in general comparable in strength to the hopping and, unless properly compensated, will influence the dynamics for several excitations. They are thus important for the determination of the many-body ground state properties. The detailed study of such effects will be the subject of future work. Therefore, the dynamics of two excitations is accounted for by a modified homogenous magnetic gauge field. Finally, for three excitations, all virtual processes are forbidden and we recover a time-reversal symmetric dynamics.

For atoms on a honeycomb array, the situation can no longer be described by a homogeneous magnetic field. As shown in Fig. 6(b), the Peierls phase $\varphi$ resulting from the (a)

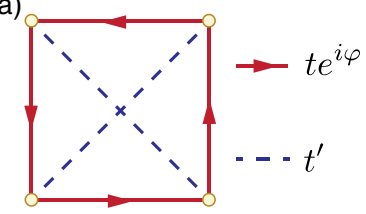

(b)

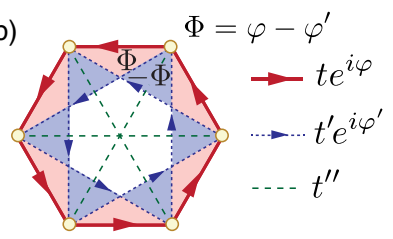

FIG. 6. Flux pattern resulting from the complex hopping for plaquettes of various geometries. (a) Square geometry. The effective Hamiltonian approach yields $t e^{i \varphi}=t_{b}+i w^{2} /(\mu \sqrt{2})$ and $t^{\prime}=t_{b} / 2^{3 / 2}-2 w^{2} / \mu$. In this case, the flux $4 \varphi$ through the square corresponds to a homogeneous magnetic field. (b) Honeycomb geometry. Here, $t e^{i \varphi}=t_{b}+3 w^{2} /(4 \sqrt{3} \mu) e^{i \pi / 3}, t^{\prime} e^{i \varphi^{\prime}}=$ $t_{b} / 3^{3 / 2}+139 w^{2} /(108 \mu) e^{2 i \pi / 3}$, and $t^{\prime \prime}=t_{b} / 8-4 w^{2} /(3 \sqrt{3} \mu)$. The flux pattern is well described as a homogeneous magnetic field with flux $6 \varphi$ through the honeycomb in combination with an alternating flux $\Phi=\varphi-\varphi^{\prime}$ through the red and blue triangles. 
nearest-neighbor hopping gives rise to a homogenous magnetic field with total flux $6 \varphi$. In addition, considering the next-nearest-neighbor coupling introduces a second Peierls phase $\varphi^{\prime}$. The combination of the two phases leads to an alternating flux pattern. Such a pattern has been previously discussed in connection to the Haldane model on a honeycomb lattice [50] and provides an intuitive explanation for the appearance of nontrivial Chern numbers with $C= \pm 1$ reported in Refs. [36,38]. For a lattice geometry consisting of many plaquettes, the Peierls phase $\varphi$ for the nearest-neighbor hopping would now vanish by symmetry, whereas the second Peierls phase $\varphi^{\prime}$ of nextnearest hopping remains finite. We would thus be able to observe chiral edge states in the single-particle regime. In Ref. [38] we obtained these chiral edge states by analyzing the band structure of the system and computing the associated Chern numbers for the $V$-structure levels scheme. The perturbative approach presented here provides more intuition on the link between the honeycomb configuration and the Haldane model.

\section{CONCLUSION}

We have experimentally demonstrated the spin-orbit coupling naturally present in dipolar exchange interactions by observing the characteristic chiral motion of an excitation in a minimal setup of three Rydberg atoms. A simple explanation of this chiral motion is achieved in the perturbative regime, where the spin-orbit coupling gives rise to Peierls phases describing a homogenous magnetic field through the triangle. Notably, the Peierls phase depends on the occupation of neighboring sites and therefore naturally gives rise to a dynamical gauge field. Especially, we have demonstrated in our minimal setup that these density-dependent Peierls phases can be interpreted as particles with an anyonic exchange statistics. This minimal setup can be extended to one-dimensional anyonHubbard and lattice-gauge field models, which will be discussed elsewhere [51]. By varying the spatial arrangement, we engineer geometry-dependent Peierls phases and explore theoretically configurations beyond the triangle. In particular, for the honeycomb plaquette, we show that at the single-particle level and in the perturbative approach, our system shows the same couplings as those of the celebrated Haldane model, which is characterized by a nontrivial topological band structure. This leads to an intriguing open question, whether the combination of such topological band structures with the strong interactions between the bosonic particles can lead to the experimental observation of integer or fractional Chern insulators [52,53].

\section{ACKNOWLEDGMENTS}

We thank Hannah Williams for discussions and for her careful reading of the manuscript. This project has received funding from the European Union's Horizon 2020 research and innovation program under Grant Agreement No. 817482 (PASQuanS), by the Région Île-de-France in the framework of DIM SIRTEQ (project CARAQUES), by the IXCORE-Fondation pour la Recherche as well as the French-German collaboration for joint projects in NLE Sciences funded by the Deutsche Forschungsgemeinschaft (DFG) and the Agence National de la Recherche (ANR, project RYBOTIN). M.F. is supported by the Deutsche Forschungsgemeinschaft (DFG) through SFB TR185, Project No. 277625399. H. P. B. is supported by the European Union under the ERC consolidator grant SIRPOL (Grant No. 681208). H. P. B., A. B., and M.F. thank the KITP for hospitality. This research was also supported in part by the National Science Foundation under Grant No. NSF PHY-1748958.

\section{APPENDIX A: NUMERICAL SIMULATION OF THE DYNAMICS}

Here, we present numerical simulations of the dynamics of the excitations in the triangle, including all the Zeeman sublevels of the $60 S_{1 / 2}$ and $60 P_{3 / 2}$ manifolds.

We first describe the role of the different exchange terms of the dipole-dipole interaction of Eq. (1) on the various Zeeman states (Fig. 7). The terms $\hat{d}_{i}^{+} \hat{d}_{j}^{-}, \hat{d}_{i}^{-} \hat{d}_{j}^{+}, \hat{d}_{i}^{+} \hat{d}_{j}^{+}$, and $\hat{d}_{i}^{-} \hat{d}_{j}^{-}$keep the system inside the $V$ structure consisting of the three states $\{|0\rangle,|+\rangle,|-\rangle\}$. On the contrary, the $\hat{d}_{i}^{z} \hat{d}_{j}^{z}$ term couples Zeeman states outside the $V$ structure. The effect of this last term is, however, inhibited thanks to the electric and magnetic fields, which energetically isolate the $V$ structure. In this case, the hopping dynamics is described by the Hamiltonian

$$
\begin{aligned}
H= & \sum_{i \neq j}\left(a_{i}^{\dagger} b_{i}^{\dagger}\right)\left(\begin{array}{cc}
-t_{a} & w e^{-i 2 \phi_{i j}} \\
w e^{i 2 \phi_{i j}} & -t_{b}
\end{array}\right)\left(\begin{array}{l}
a_{j} \\
b_{j}
\end{array}\right) \\
& +\sum_{i} \frac{\mu}{2}\left(n_{i}^{a}-n_{i}^{b}\right)+H_{\mathrm{vdW}},
\end{aligned}
$$

where the two bosonic operators $a_{i}^{\dagger}$ and $b_{i}^{\dagger}$ on site $i$ are defined by $a_{i}^{\dagger}|0\rangle=|+\rangle_{i}$ and $b_{i}^{\dagger}|0\rangle=|-\rangle_{i}$. The hopping amplitudes $t_{a, b}$ are related to the dipole matrix elements by

$$
t_{a, b}=\frac{\left|\left\langle \pm\left|\hat{d}_{+}\right| 0\right\rangle\right|^{2}}{8 \pi \epsilon_{0} r_{i j}^{3}}, \quad w=\frac{3\left\langle+\left|\hat{d}_{+}\right| 0\right\rangle\left\langle 0\left|\hat{d}_{-}\right|-\right\rangle}{8 \pi \epsilon_{0} r_{i j}^{3}} .
$$

The term $H_{\mathrm{vdW}}$ includes the van der Waals interactions between the Rydberg levels (typically around $70 \mathrm{kHz}$ ), which are negligible with respect to the hopping amplitudes. However, we do include it for the quantitative comparison between theory and experimental results.

To isolate the $V$ structure, we apply dc magnetic and electric fields perpendicular to the triangle. The magnetic 


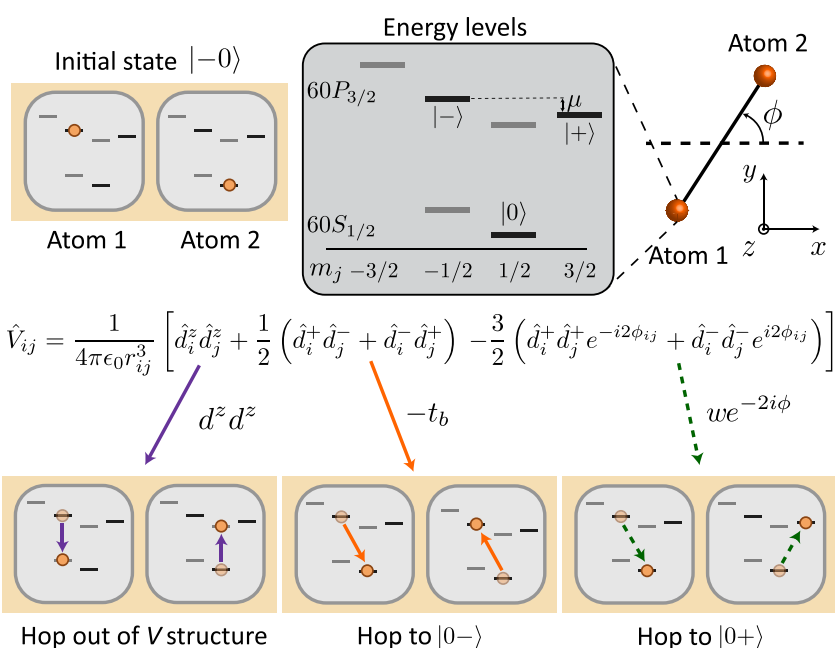

FIG. 7. Rydberg levels in a $V$ structure and hopping processes. We consider two atoms as shown on top of the figure, with their six Zeeman sublevels and focus on the $V$ structure highlighted in black. Starting from the initial state $|-0\rangle$, the dipole-dipole interaction in the case of a quantization axis perpendicular to the atom array induces three types of hopping. The first term of the dipole-dipole interaction makes the system leave the $V$ structure. The two other terms are the direct (solid arrow) and the complex (dashed arrow) hoppings, mentioned in the main text.

field of $8.5 \mathrm{G}$ lifts the degeneracy of the Zeeman sublevels of a single atom. However, the pair state $|--\rangle$ is still degenerate with $\left|60 P_{3 / 2}, m_{j}=-3 / 2,60 P_{3 / 2}, m_{j}=1 / 2\right\rangle$. To avoid leakage to this state due to the resonant interaction, we lift the degeneracy by additionally applying an electric field. We choose $E_{z}=0.4 \mathrm{~V} / \mathrm{cm}$, for which the static dipole moment induced by the electric field is still small. Isolating Rydberg manifolds prevents one from using short interatomic distances where Rydberg levels get intermixed. On the other hand, strong interactions and thus, fast dynamics are necessary to neglect the decay of the Rydberg levels and the motion of atoms. For our experiment, an interatomic distance of $11 \mu \mathrm{m}$ is a good trade-off. All parameters are optimized under the constraint that the condition for chiral propagation is fulfilled.

As seen in the main text, the dynamics of the excitations in the triangle can be qualitatively understood by considering only the exchange interactions between the levels of the $V$ structure, see Fig. 2. Since the isolation of the $V$ structure is in practice not perfect, we perform simulations including all Zeeman sublevels of the $60 S_{1 / 2}$ and $60 P_{3 / 2}$ manifolds. Considering the Rydberg states outside these two manifolds results in van der Waals interactions between the atoms, which we include in the simulation. This interaction has, however, a negligible influence on the dynamics for the parameters used in the experiment. The strengths of both the resonant and van der Waals interactions are calculated in the presence of the applied electric and magnetic fields using our open source calculator [44].
The simulation starts with a triangle where each atom is in the $|0\rangle$ state. As a first step, we simulate the preparation of the $|-\rangle$ excitations using the addressing beam inducing a local light shift of typically $6 \mathrm{MHz}$, and the microwave pulse. We take into account the van der Waals and exchange interactions between the atoms during the preparation. After prediagonalizing the single-atom Hamiltonians describing the interaction of the atoms with the applied static fields, we introduce the microwave couplings. The microwave couples the Stark- and Zeeman-shifted states of the $60 S_{1 / 2}$ manifold to the $60 P_{3 / 2}$ manifold. As mentioned in Sec. III, we apply a light shift to one atom and tune the frequency of the microwave to be resonant with the transition to the $|-\rangle$ excitation. The computations are performed in the rotating frame within the rotating-wave approximation. The simulation of the preparation process indicates leakage to other states outside the $V$ structure, on the order of 5\%. Presumably, this leakage could be reduced using optimal control. As a second step, we simulate the time evolution of the prepared state under the influence of the dipolar exchange interaction.

We take into account experimental imperfections by sampling over 500 different realizations of the initial configuration of the triangles. First, we take for the probability for lattice vacancies (due to missing atoms or errors in the stimulated Raman adiabatic passage process) the measured value 0.17 . Second, we consider shot-to-shot fluctuations of the positions of the atoms in their tweezers, which results in varying hopping strengths. Importantly, due to these fluctuations, the atoms can also be positioned in such a way that the interatomic axis is not exactly perpendicular to the quantization axis. In this case, the dipolar interaction can change the magnetic quantum number by one, provoking additional leakage to states outside the $V$ structure. These experimental imperfections are responsible for the observed damping of the dynamics. Finally, detection errors are included through a Monte Carlo sampling of the numerical results [34,54]. In the simulations we account for the fact that the detection scheme does not distinguish between states other than $|0\rangle$ by computing the probabilities $P_{|100\rangle}, P_{|010\rangle}$, and $P_{|001\rangle}$ as measured in the experiment. Note that the preparation of a state with two excitations is experimentally challenging and prone to additional errors. Therefore, we have scaled vertically the theory curve shown in Fig. 4(b) by a factor 0.8 .

\section{APPENDIX B: MAPPING ONTO AN ANYONIC PROBLEM}

\section{Formal mapping}

Here, we demonstrate that the excitations on a triangle, described by a Hamiltonian with the density-dependent Peierls phases, can be understood as a system of hard-core Abelian anyons with a nontrivial phase under exchange. We start from the Hamiltonian (4) 
$H_{\mathrm{eff}}^{\mathrm{many}}=-t \sum_{i=1}^{3}\left[e^{i \varphi\left(1-n_{i+2}\right)} b_{i+1}^{\dagger} b_{i}+\Delta b_{i+1}^{\dagger} b_{i} n_{i+2}+\right.$ H.c. $]$,

with $n_{i}=b_{i}^{\dagger} b_{i}$. The excitations are described by the bosonic creation (annihilation) operators $b_{i}^{\dagger}\left(b_{i}\right)$, respectively, with $\left[b_{i}, b_{j}^{\dagger}\right]=0$ and $\left[b_{i}, b_{j}\right]=0$ for $i \neq j$. The hardcore constraint is most conveniently accounted for by the anticommutation relations $\left\{b_{i}, b_{i}^{\dagger}\right\}=1$ and $\left\{b_{i}, b_{i}\right\}=0$.

In order to map the Hamiltonian to Abelian anyons, we define the new modes $B_{n}$ by the unitary transformation

$$
\begin{aligned}
B_{1}^{\dagger} & \equiv e^{-i \varphi\left(3-n_{2}-2 n_{3}\right)} b_{1}, \\
B_{2}^{\dagger} & \equiv e^{-i \varphi\left(1+2 n_{1}-3 n_{3}\right)} b_{2}, \\
B_{3}^{\dagger} & \equiv e^{-i \varphi\left(n_{1}-1\right)} b_{3} .
\end{aligned}
$$

Under this transformation, the Hamiltonian (4) now takes the simple form

$H=-t \sum_{i=1}^{3}\left[B_{i+1}^{\dagger} B_{i}+\Delta B_{i+1}^{\dagger} B_{i}\left(1-B_{i+2}^{\dagger} B_{i+2}\right)+\right.$ H.c. $]$

which does not feature the density-dependent Peierls phases. The second term in Eq. (B3) describes a conventional correlated hopping where the (real) couplings depend on the number of particles in the new modes. The influence of the Peierls phases is now hidden in the nontrivial commutation relations of the modes $B_{n}$, which can be shown to obey

$$
\begin{aligned}
\left\{B_{n}, B_{n}^{\dagger}\right\} & =1, \\
\left\{B_{n}, B_{n}\right\} & =0, \\
B_{n} B_{m} & =e^{3 i \varphi \operatorname{sign}(n-m)} B_{m} B_{n}, \\
B_{n}^{\dagger} B_{m} & =e^{-3 i \varphi \operatorname{sign}(n-m)} B_{m} B_{n}^{\dagger},
\end{aligned}
$$

for $n \neq m$. Here, $\operatorname{sign}(x)=1$ for $x \geq 0$ and $\operatorname{sign}(x)=-1$ for $x<0$. This is the algebra of Abelian anyons in one dimension with statistical angle $3 \varphi$ and infinite, repulsive on-site interaction (a hard-core constraint) [43]. For $\varphi=0$ $(\varphi=\pi / 3)$ we recover hard-core bosons (fermions). However, for the flux $\pi / 2$ with $\varphi=\pi / 6$ one finds the nontrivial "semionic" commutation relations [55]

$$
B_{1} B_{2}=-i B_{2} B_{1} \quad \text { and } \quad B_{1}^{\dagger} B_{2}=i B_{2} B_{1}^{\dagger} \text {, etc., }
$$

that describe particles "halfway" between bosons and fermions.

\section{Interpretation of the dynamics on a triangle}

The dynamical behavior of one or two excitations $\left(b_{i}^{\dagger}\right)$ observed in the experiment can now be interpreted as follows in the anyonic picture $\left(B_{n}^{\dagger}\right)$. Because of the implicit particle-hole transformation in (B2), a single excitation $b_{i}^{\dagger}$ corresponds to a triangle occupied by two anyons $B_{n}^{\dagger}$. The chiral motion of a single excitation due to the magnetic field in (3) therefore maps onto the chiral motion of a hole flanked by two anyons in (B3). However, in the anyonic picture, the phase that induces chiral motion is not due to a magnetic field [which is absent in (B3)], but rather is a consequence of the statistical phase collected by two anyons that exchange places. Similarly, the symmetric motion in the case of two excitations $b_{i}^{\dagger}$ corresponds to the nonchiral dynamics of a single anyon $B_{n}^{\dagger}$ subject to (B3), i.e., without magnetic field.

[1] I. M. Georgescu, S. Ashhab, and F. Nori, Quantum Simulation, Rev. Mod. Phys. 86, 153 (2014).

[2] E. J. Bergholtz and Z. Liu, Topological Flat Band Models and Fractional Chern Insulators, Int. J. Mod. Phys. B 27, 1330017 (2013).

[3] N. R. Cooper, J. Dalibard, and I. B. Spielman, Topological Bands for Ultracold Atoms, Rev. Mod. Phys. 91, 015005 (2019).

[4] D. R. Hofstadter, Energy Levels and Wave Functions of Bloch Electrons in Rational and Irrational Magnetic Fields, Phys. Rev. B 14, 2239 (1976).

[5] D. Jaksch and P. Zoller, Creation of Effective Magnetic Fields in Optical Lattices: The Hofstadter Butterfly for Cold Neutral Atoms, New J. Phys. 5, 56 (2003).

[6] N. Goldman, G. Juzeliūnas, P. Öhberg, and I. B. Spielman, Light-Induced Gauge Fields for Ultracold Atoms, Rep. Prog. Phys. 77, 126401 (2014).

[7] J. Dalibard, F. Gerbier, G. Juzeliūnas, and P. Öhberg, Colloquium: Artificial Gauge Potentials for Neutral Atoms, Rev. Mod. Phys. 83, 1523 (2011).

[8] V. Galitski and I. B. Spielman, Spin-Orbit Coupling in Quantum Gases, Nature (London) 494, 49 (2013).

[9] H. Zhai, Degenerate Quantum Gases with Spin-Orbit Coupling: A Review, Rep. Prog. Phys. 78, 026001 (2015).

[10] M. Aidelsburger, S. Nascimbene, and N. Goldman, Artificial Gauge Fields in Materials and Engineered Systems, C.R. Phys. 19, 394 (2018).

[11] M. Aidelsburger, M. Atala, S. Nascimbène, S. Trotzky, Y.-A. Chen, and I. Bloch, Experimental Realization of Strong Effective Magnetic Fields in an Optical Lattice, Phys. Rev. Lett. 107, 255301 (2011).

[12] A. R. Kolovsky, Creating Artificial Magnetic Fields for Cold Atoms by Photon-Assisted Tunneling, Europhys. Lett. 93, 20003 (2011). 
[13] J. Struck, C. Ölschläger, M. Weinberg, P. Hauke, J. Simonet, A. Eckardt, M. Lewenstein, K. Sengstock, and P. Windpassinger, Tunable Gauge Potential for Neutral and Spinless Particles in Driven Optical Lattices, Phys. Rev. Lett. 108, 225304 (2012).

[14] G. Jotzu, M. Messer, R. Desbuquois, M. Lebrat, T. Uehlinger, D. Greif, and T. Esslinger, Experimental Realization of the Topological Haldane Model with Ultracold Fermions, Nature (London) 515, 237 (2014).

[15] M. Mancini, G. Pagano, G. Cappellini, L. Livi, M. Rider, J. Catani, C. Sias, P. Zoller, M. Inguscio, M. Dalmonte, and L. Fallani, Observation of Chiral Edge States with Neutral Fermions in Synthetic Hall Ribbons, Science 349, 1510 (2015).

[16] B. K. Stuhl, H.-I. Lu, L. M. Aycock, D. Genkina, and I. B. Spielman, Visualizing Edge States with an Atomic Bose Gas in the Quantum Hall Regime, Science 349, 1514 (2015).

[17] T. Chalopin, T. Satoor, A. Evrard, V. Makhalov, J. Dalibard, R. Lopes, and S. Nascimbène, Exploring the Topology of a Quantum Hall System at the Microscopic Level, arXiv: 2001.01664.

[18] P. Roushan et al., Chiral Ground-State Currents of Interacting Photons in a Synthetic Magnetic Field, Nat. Phys. 13, 146 (2017).

[19] T. Ozawa, H. M. Price, A. Amo, N. Goldman, M. Hafezi, L. Lu, M. C. Rechtsman, D. Schuster, J. Simon, O. Zilberberg, and I. Carusotto, Topological Photonics, Rev. Mod. Phys. 91, 015006 (2019).

[20] Y. Liu, X. Chen, and Y. Xu, Topological Phononics: From Fundamental Models to Real Materials, Adv. Funct. Mater. 30, 1904784 (2019).

[21] M. Saffman, T. G. Walker, and K. Mølmer, Quantum Information with Rydberg Atoms, Rev. Mod. Phys. 82, 2313 (2010).

[22] H. Weimer, M. Müller, I. Lesanovsky, P. Zoller, and H. P. Büchler, A Rydberg Quantum Simulator, Nat. Phys. 6, 382 (2010).

[23] Y. Miroshnychenko, W. Alt, I. Dotsenko, L. Förster, M. Khudaverdyan, A. Rauschenbeutel, and D. Meschede, Precision Preparation of Strings of Trapped Neutral Atoms, New J. Phys. 8, 191 (2006).

[24] D. Barredo, S. de Léséleuc, V. Lienhard, T. Lahaye, and A. Browaeys, An Atom-by-Atom Assembler of Defect-Free Arbitrary Two-Dimensional Atomic Arrays, Science 354, 1021 (2016).

[25] M. Endres, H. Bernien, A. Keesling, H. Levine, E. R. Anschuetz, A. Krajenbrink, C. Senko, V. Vuletic, M. Greiner, and M.D. Lukin, Atom-by-Atom Assembly of Defect-Free One-Dimensional Cold Atom Arrays, Science 354, 1024 (2016).

[26] H. Kim, W. Lee, H. Lee, H. Jo, Y. Song, and J. Ahn, In situ Single-Atom Array Synthesis Using Dynamic Holographic Optical Tweezers, Nat. Commun. 7, 13317 (2016).

[27] D. Barredo, V. Lienhard, S. de Léséleuc, T. Lahaye, and A. Browaeys, Synthetic Three-Dimensional Atomic Structures Assembled Atom by Atom, Nature (London) 561, 79 (2018).

[28] D. O. de Mello, D. Schäffner, J. Werkmann, T. Preuschoff, L. Kohfahl, M. Schlosser, and G. Birkl, Defect-Free Assembly of 2D Clusters of More than 100 Single-Atom Quantum Systems, Phys. Rev. Lett. 122, 203601 (2019).
[29] A. Browaeys, D. Barredo, and T. Lahaye, Experimental Investigations of Dipole-Dipole Interactions between a Few Rydberg Atoms, J. Phys. B 49, 152001 (2016).

[30] H. Labuhn, D. Barredo, S. Ravets, S. de Léséleuc, Macrì, T. Lahaye, and A. Browaeys, Tunable Two-Dimensional Arrays of Single Rydberg Atoms for Realizing Quantum Ising Models, Nature (London) 534, 667 (2016).

[31] H. Bernien, S. Schwartz, A. Keesling, H. Levine, A. Omran, H. Pichler, S. Choi, A. S. Zibrov, M. Endres, M. Greiner, V. Vuletic, and M. D. Lukin, Probing Many-Body Dynamics on a 51-Atom Quantum Simulator, Nature (London) 551, 579 (2017).

[32] H. Kim, Y. Park, K. Kim, H.-S. Sim, and J. Ahn, Detailed Balance of Thermalization Dynamics in RydbergAtom Quantum Simulators, Phys. Rev. Lett. 120, 180502 (2018).

[33] D. Barredo, H. Labuhn, S. Ravets, T. Lahaye, A. Browaeys, and C. S. Adams, Coherent Excitation Transfer in a Spin Chain of Three Rydberg Atoms, Phys. Rev. Lett. 114, 113002 (2015).

[34] S. de Léséleuc, V. Lienhard, P. Scholl, D. Barredo, S. Weber, N. Lang, H. P. Büchler, T. Lahaye, and A. Browaeys, Observation of a Symmetry-Protected Topological Phase of Interacting Bosons with Rydberg Atoms, Science 365, 775 (2019).

[35] S. V. Syzranov, M. L. Wall, V. Gurarie, and A. M. Rey, Spin-Orbital Dynamics in a System of Polar Molecules, Nat. Commun. 5, 5391 (2014).

[36] D. Peter, N. Y. Yao, N. Lang, S. D. Huber, M. D. Lukin, and H. P. Büchler, Topological Bands with a Chern Number $C=2$ by Dipolar Exchange Interactions, Phys. Rev. A 91, 053617 (2015).

[37] M. Kiffner, E. O'Brien, and D. Jaksch, Topological Spin Models in Rydberg Lattices, Appl. Phys. B 123, 46 (2017).

[38] S. Weber, S. de Léséleuc, V. Lienhard, D. Barredo, T. Lahaye, A. Browaeys, and H. P. Büchler, Topologically Protected Edge States in Small Rydberg Systems, Quantum Sci. Technol. 3, 044001 (2018).

[39] U.-J. Wiese, Ultracold Quantum Gases and Lattice Systems: Quantum Simulation of Lattice Gauge Theories, Ann. Phys. (Berlin) 525, 777 (2013).

[40] F. Görg, K. Sandholzer, J. Minguzzi, R. Desbuquois, M. Messer, and T. Esslinger, Realization of DensityDependent Peierls Phases to Engineer Quantized Gauge Fields Coupled to Ultracold Matter, Nat. Phys. 15, 1161 (2019).

[41] L. W. Clark, B. M. Anderson, L. Feng, A. Gaj, K. Levin, and C. Chin, Observation of Density-Dependent Gauge Fields in a Bose-Einstein Condensate Based on Micromotion Control in a Shaken Two-Dimensional Lattice, Phys. Rev. Lett. 121, 030402 (2018).

[42] C. Schweizer, F. Grusdt, M. Berngruber, L. Barbiero, E. Demler, N. Goldman, I. Bloch, and M. Aidelsburger, Floquet Approach to $Z_{2}$ Lattice Gauge Theories with Ultracold Atoms in Optical Lattices, Nat. Phys. 15, 1168 (2019).

[43] E. Fradkin, Field Theories of Condensed Matter Systems (Addison-Wesley, Redwood City, CA, 1991). 
[44] S. Weber, C. Tresp, H. Menke, A. Urvoy, O. Firstenberg, H.P. Büchler, and S. Hofferberth, Calculation of Rydberg Interaction Potentials, J. Phys. B 50, 133001 (2017).

[45] S. de Léséleuc, D. Barredo, V. Lienhard, A. Browaeys, and T. Lahaye, Optical Control of the Resonant Dipole-Dipole Interaction between Rydberg Atoms, Phys. Rev. Lett. 119, 053202 (2017).

[46] J.-X. Zhu and Z. D. Wang, Topological Effects Associated with Fractional Statistics in One-Dimensional Mesoscopic Rings, Phys. Rev. A 53, 600 (1996).

[47] A. Kundu, Exact solution of double $\delta$ function Bose Gas through an Interacting ANYON Gas, Phys. Rev. Lett. 83, 1275 (1999).

[48] T. Keilmann, S. Lanzmich, I. McCulloch, and M. Roncaglia, Statistically Induced Phase Transitions and Anyons in 1D Optical Lattices, Nat. Commun. 2, 361 (2011).

[49] S. Greschner and L. Santos, Anyon Hubbard Model in OneDimensional Optical Lattices, Phys. Rev. Lett. 115, 053002 (2015).
[50] F. D. M. Haldane, Model for a Quantum Hall Effect without Landau Levels: Condensed-Matter Realization of the Parity Anomaly, Phys. Rev. Lett. 61, 2015 (1988).

[51] (to be published).

[52] Y.-F. Wang, Z.-C. Gu, C.-D. Gong, and D. N. Sheng, Fractional Quantum Hall Effect of Hard-Core Bosons in Topological Flat Bands, Phys. Rev. Lett. 107, 146803 (2011).

[53] Y.-F. Wang, H. Yao, C.-D. Gong, and D. N. Sheng, Fractional Quantum Hall Effect in Topological Flat Bands with Chern Number Two, Phys. Rev. B 86, 201101(R) (2012).

[54] S. de Léséleuc, D. Barredo, V. Lienhard, A. Browaeys, and T. Lahaye, Analysis of Imperfections in the Coherent Optical Excitation of Single Atoms to Rydberg States, Phys. Rev. A 97, 053803 (2018).

[55] A. Zee, Semionics: A Theory of Superconductivity Based on Fractional Quantum Statistics, in Proceedings of the 25th International Conference on High-Energy Physics, Singapore, 1990, edited by K. K. Phua and Y. Yamaguchi (World Scientific Publishing Co Pte Ltd, Singapore, 1991), Vol. I, pp. 206-212. 